
\title{
3 Research Square \\ Reconstruction of Epidural Fat to Prevent Epidural Fibrosis after Laminectomy in Rabbits
}

\section{Xiangsheng Liu}

Fifth People's Hospital of Shanghai Fudan University

\section{Feifei Zhang}

Shanghai Xuhui dahua Hospital

\section{Linli Li}

Fifth People's Hospital of Shanghai Fudan University

\section{Yiqun $\mathrm{He}$}

Fifth People's Hospital of Shanghai Fudan University

\section{Youhai Dong ( $\nabla$ youhaidong1964@163.com )}

Fifth People's Hospital of Shanghai Fudan University https://orcid.org/0000-0001-6841-6262

\section{Research article}

Keywords: Laminectomy; tissue-engineered fat; epidural fibrosis; epidural fat; adipogenesis; adipogenic mesenchymal stem cells; extracellular matrix gel

Posted Date: March 22nd, 2021

DOI: https://doi.org/10.21203/rs.3.rs-314557/v1

License: (c) (i) This work is licensed under a Creative Commons Attribution 4.0 International License. Read Full License

Version of Record: A version of this preprint was published at Tissue Engineering Part A on September 25th, 2021. See the published version at https://doi.org/10.1089/ten.tea.2021.0097. 


\section{Abstract}

Background: Laminectomy can effectively decompress the spinal cord and expand the vertebral canal. However, the fibrosis that appears may cause adherence and recompression of the spinal cord or/ and nerve root, which may cause Failed Back Syndrome (FBS) and make the re-exposure process more difficult. Reconstruction of the epidural fat may be an ideal method to achieve satisfactory results.

Methods: Thirty-six New Zealand rabbits were randomly divided into three groups: control, ECM, and ECM+aMSCs groups. Saline, ECM gel, and ECM+aMSC complex were placed respectively at the fifth lumbar vertebrate of the rabbits. Epidural fat and fibrosis formation were detected by MRI and histologically at the $4^{\text {th }}, 8^{\text {th }}$, and $12^{\text {th }}$ weeks. Quantitative RT-PCR was used to detect the expression of IL6 and TGF- $\beta$.

Results: MRI and Oil Red 0 staining revealed epidural fat formation at the $12^{\text {th }}$ week in the ECM+aMSCs group. H\&E staining showed that the numbers of fibroblasts in the ECM gel and ECM+aMSCs groups were less than the control group at the $4^{\text {th }}$ and $8^{\text {th }}$ weeks $(P<0.05)$. Masson's trichrome staining showed that the proportion of collagen fibers in the ECM gel and ECM+aMSCs group was lower than the control group $(P<0.05)$. Quantitative RT-PCR showed the expressions of TGF- $\beta$ and IL- 6 were lower in the ECM gel and ECM+aMSCs group than the control group $(P<0.05)$ at the $4^{\text {th }}$ week, but higher at the $8^{\text {th }}$ week.

Conclusion: We successfully reconstructed the epidural fat with ECM gel and aMSC complex; additionally, IL- 6 and TGF- $\beta$ cytokines were lower at early stage after laminectomy.

\section{Background}

Total laminectomy is a classic surgical method for the treatment of spinal diseases. After laminectomy, fibrosis appears at epidural adhering and recompresses the spinal cord or/ and nerve root, plays an important role in the recurrence of back and leg pain after spinal surgery called Failed Back Syndrome $(\mathrm{FBS})^{1}$, and makes the re-exposure of the dura without durotomy more difficult. Fibroblasts play an important role in the formation of epidural fibrosis due to the proliferation, migration, and secretion of numerous collagen fibers ${ }^{2,3}$. The secretion of inflammatory cytokines, such as IL- 6 and TGF- $\beta$, can promote the migration, proliferation, and differentiation of fibroblasts, which results in the activation of fibroblasts producing excessive extracellular fiber tissues ${ }^{4,5}$. Many drugs have been tested to reduce epidural fibrosis, such as non-steroidal anti-inflammatory drugs, mitomycin C/5-FU, and hyaluronic acid, but the long-term effect remains unsatisfactory ${ }^{6-8}$. The epidural fat tissue acts as a physiological barrier between the dural sac and the fibrous tissue reduces the problem of epidural fiber adhesion ${ }^{9}$. Many studies have attempted to reconstruct epidural fat tissue to prevent epidural fibrosis using tissue engineering techniques. The biomaterials used for the epidural fat reconstruction were mainly solid membrane, such as PLGA membrane, amniotic membrane, Adcon- $L$, and so on ${ }^{10,11}$. These solid membranes have several shortcomings for epidural fat reconstruction, such as their rigidity (which may cause compression again) and lack of amorphous state (as it cannot conform to the defect shape). 
The extracellular matrix (ECM) is a non-cellular three-dimensional macromolecular network composed of collagens, proteoglycans, elastin, laminins, and several other glycoproteins. The ECM not only forms a complex network into which cells reside in all tissues and organs, but also provides signals to cells to regulate their survival, growth, migration, and differentiation. ECM gel is in the fluid state at $2^{\circ} \mathrm{C}-8^{\circ} \mathrm{C}$ and turns into the gel state at $20^{\circ} \mathrm{C}-40^{\circ} \mathrm{C}$. Its thermoresponsive properties makes it feasible to be mixed with stem cells and injected into the defect sites at $2^{\circ} \mathrm{C}-8^{\circ} \mathrm{C}$, and then act as a scaffold for the cells in the in vivo environment.

In this study, we constructed the tissue-engineered fat using ECM gel and rabbit umbilical cord Whorton's Jelly-derived mesenchymal stem cells (WJ-MSCs), and then examined whether the tissue-engineered fat could reconstruct the epidural fat and reduce epidural fibrosis in the laminectomy rabbits.

\section{Materials And Methods}

\section{Isolation and culture of WJ-MSCs}

The protocol for isolation and culture of rabbit umbilical cord mesenchymal stem cells was the same as that published in our previous paper ${ }^{12}$. Briefly, pregnant rabbits $\left(27^{\text {th }}\right.$ day of pregnancy) were narcotized using 3\% pentobarbital sodium (Germany Merck Biotechnology Co., Ltd.). The umbilical cords were harvested under sterile conditions and dissected to expose the Wharton's Jelly (WJ). The Wharton's Jelly was cut into small parts and digested with $1 \mathrm{mg} / \mathrm{ml}$ collagenase II (Sigma-Aldrich) at $37^{\circ} \mathrm{C}$ for 30 minutes. The digestion was terminated by low glucose Dulbecco's Modified Eagle's Medium (LG-DMEM, Gibco, USA), containing 10\% fetal bovine serum (FBS, Biological industries, Israel) and $100 \mathrm{U} / \mathrm{mL}$ of penicillin with $100 \mathrm{mg} / \mathrm{ml}$ streptomycin (Gibco, USA). The digested solution was centrifuged, the cells in the sediment were resuspended using the complete culture medium, and then plated on Petri dishes at a density of $4 \times 10^{4} / \mathrm{cm}^{2}$ in an incubator at $37^{\circ} \mathrm{C}$ under a $5 \% \mathrm{CO}_{2}$ atmosphere. Media replacement was performed every 72 hours and the cells were allowed to grow until cell cultures reached $70 \%$ to $80 \%$ confluence.

\section{Construction of ECM gel and adipogenic WJ-MSC complex}

Cells at passage 6 were used for the following experiments. Cells were cultured in adipogenic media inducing solution A (dexamethasone $1 \mu \mathrm{M}$, IBMX $0.5 \mathrm{mM}$, insulin $10 \mu \mathrm{g} / \mathrm{ml}$, and indomethacin $200 \mu \mathrm{g}$ added to complete medium) for 72 hours, and then replaced with solution $B(10 \mu \mathrm{g} / \mathrm{ml}$ insulin added to the complete medium) for another 72 hours. After 15 days, the success of adipogenic induction was identified by Oil Red $\mathrm{O}$ staining. Then the adipogenic WJ-MSCs (aMSCs) were digested and resuspended in the complete culture media at $4^{\circ} \mathrm{C}$ at a density of $1 \times 10^{6} \mathrm{cells} / \mathrm{ml}$. The tissue-engineered fat complex was prepared by mixing with ECM gel (Sigma-Aldrich) and the cell suspension with the ratio of 1:1. The complex was kept at $4^{\circ} \mathrm{C}$ until use (the time period was less than 1 hour).

\section{Experimental animals}


Thirty-six New Zealand rabbits were randomly divided into three groups: control group, ECM group, and ECM+aMSCs group. The treatments were as follows: Control group was treated with $1 \mathrm{ml}$ saline after laminectomy, the ECM group was treated with $1 \mathrm{ml} \mathrm{ECM} \mathrm{gel,} \mathrm{and} \mathrm{the} \mathrm{ECM+aMSCs} \mathrm{group} \mathrm{was} \mathrm{treated} \mathrm{with}$ $1 \mathrm{ml}$ of the tissue-engineered complex with ECM gel as scaffold and aMSCs as the seed cells.

The New Zealand rabbits weighed approximately 3.0-3.5 kg and were injected intraperitoneally with $3 \%$ pentobarbital sodium (Germany Merck Biotechnology Co., Ltd.) at a dose of 1-1.5 ml/kg for anesthesia. Next, the rabbit's back was depilated and disinfected with iodophor. The skin at the L5 vertebrate was cut, the subcutaneous tissue was exposed, and the superficial fascia and supraspinous ligament was incised to expose the L5 laminae. Then the spinous process and laminae were removed by rongeur, and the epidural fat was carefully eliminated to expose the spinal dura sac. Then the corresponding groups were injected with corresponding materials with injection syringe. The wounds were closed with 4-0 suture. Penicillin sodium $(80,000$ units $/ \mathrm{kg})$ was used to prevent infection with intramuscular injection. Then, the New Zealand rabbits were returned to the rabbit cage for routine feeding.

\section{MRI evaluation}

At the $4^{\text {th }}, 8^{\text {th }}$, and $12^{\text {th }}$ weeks after operation, three New Zealand rabbits in each group were subjected to MRI examinations to observe the formation of sagittal and transverse epidural fibers and fat at the laminectomy area.

After MRI, a little soft tissue near the laminae defect was collected in a 1.5-ml cryotube and stored in liquid nitrogen. The rabbits were euthanized by excess injection of $3 \%$ sodium pentobarbital. The L5 lumbar spine combined with the upper and lower lumbars were cut en bloc, fixed with $10 \%$ formaldehyde for 1 week, and then sent to Wuhan Google Biotechnology Co., Ltd. Shanghai Branch for decalcification treatment. EDTA (10\%) was used for decalcification for 4-8 weeks until the vertebral bone could be cut with a slicing knife.

\section{Histologic evaluation}

After decalcification, the specimens were embedded in paraffin. Hematoxylin and eosin (H\&E) staining was used to count the fibroblasts at 400X magnification (Fig. 1). The ratio of the area of blue collagen fibers to the total area of tissues in the field of view at 200X magnification was used as a measure to estimate the degree of fibrosis using Masson's trichrome staining. Oil Red $O$ staining was used to observe the formation of epidural fat.

\section{Quantitative real time polymerase chain reaction ( $q R T-P C R)$}

qRT-PCR was used to detect the relative expression of local inflammatory factor IL-6 and fibrosis factor TGF- $\beta$ in each group at each time and $\beta$-actin was used as internal reference. All tissues in the cryotubes were decomposed with the Trizol $\circledast$ reagent (Invitrogen, USA) to obtain RNA, and reverse transcriptase (Takara, Japan) was added to form cDNA. Quantitative real-time PCR (RT-PCR) was performed using the 
7900 Real-Time PCR System (Applied Biosystems) with the Power SYBR® Green PCR Master Mix (Takara, Japan) to explore the relative expression of genes. The primers (Shanghai, China) were sequenced as shown in Table 1. Relative gene expression was calculated using the equation: $\Delta \mathrm{Ct}=\mathrm{Ct}$ (Rest genes) $-\mathrm{Ct}\left(\beta\right.$-actin); $\Delta \Delta \mathrm{Ct}=\Delta \mathrm{Ct}$ (experimental groups) $-\Delta \mathrm{Ct}$ (control group); Fold change $=2^{-\Delta \Delta \mathrm{Ct}}$.

\section{Statistical Analysis}

The results of each set of experiments were repeated three times, and statistical analysis was performed using the GraphPad prism 6.0 software. The multiple $T$ test was mainly used and $P<0.05$ was considered statistically significant.

\section{Results}

\section{MRI evaluation}

MRI examination of experimental animals was performed at the $4^{\text {th }}, 8^{\text {th }}$, and $12^{\text {th }}$ weeks after surgery. At the $4^{\text {th }}$ week, we found that there were no evident fat signal at the epidural space in all groups (Fig. 2-4), and no remarkable gap between dural sac and epidural tissue. However, a small amount of epidural fibrous tissue signals was found in the ECM gel and control groups. The amount of epidural scar increased gradually over time and was the most evident at the $12^{\text {th }}$ week in all groups (Fig. 2-4). At the $8^{\text {th }}$ week, a small amount of fat signal appeared between the dural sac and epidural fibrous tissue in the ECM+aMSCs groups, and a complete and thick fat signal was observed at the $12^{\text {th }}$ week (Fig. 4).

\section{Histologic evaluation}

H\&E staining showed that the number of fibroblasts in the ECM+aMSCs groups and ECM gel groups was significantly lower than that in the control groups at the $4^{\text {th }}$ and $8^{\text {th }}$ weeks $(P<0.05)$. There was no statistically significant difference between the ECM+aMSCs group and control group at the $12^{\text {th }}$ week $(P>0.05)$ (Fig. 5).

Masson's trichrome staining showed that the collagen fiber content of each group gradually increased with time, the control groups had the highest ratio of fibrosis protein in any time period, and the ratio of fibrosis protein in $\mathrm{ECM}+\mathrm{aMSC}$ group was the lowest $(P<0.05)$ (Fig. 6).

Oil Red 0 staining showed that fat formation between the dural sac and epidural fibrous tissue in the ECM+aMSCs group was similar to the normal rabbit at the $12^{\text {th }}$ week, and the dural sac adhered to the fibrous tissue in the control group (Fig.7).

qRT-PCR results

After laminectomy, the relative expressions of inflammatory factor IL- 6 and fibrosis factor TGF- $\beta$ were further explored. It was found that the relative expressions of IL- 6 and TGF- $\beta$ in the EMC gel and 
ECM+aMSCs groups were significantly lower than those in the control group at the $4^{\text {th }}$ week $(P<0.05)$ (Fig. $\mathbf{8} ; \mathbf{4 w})$, but the expressions of them were higher at the $8^{\text {th }}$ week $(P<0.05)$ (Fig. 8; 8w). At the $12^{\text {th }}$ week, TGF- $\beta$ in the ECM gel group and IL- 6 in ECM+aMSCs group were not significantly different compared with the control group ( $P>0.05$ ) (Fig. 8; 12w).

\section{Discussion}

Tissue engineering is more widely used to restore the normal tissue via the interaction of cells, biomaterials, and factor ${ }^{13}$. Dong Youhai et al. ${ }^{14}$ used bone marrow mesenchymal stem cells with collagen sponge to construct the artificial laminae of the vertebral arch in a rabbit laminectomy model. As an easily obtained source of stem cells, aMSCs exert immunomodulatory effects for the prevention of inflammatory reaction in addition to their differentiation potential and can differentiate into adipose cells ${ }^{15,16}$. Using adipose tissue engineering technology, we reconstructed epidural fat in the laminectomy rabbits successfully and developed a physiological fat barrier at the epidural site.

MRI examination is a valuable method for the differentiation of fibrosis and fat tissue in vivo ${ }^{17}$. Clinically, the scar tissue observed from MRI is a negative sign for the FBS after lumbar surgery, and MRI is also a good method to assess the size and shape of the free-fat implanted in the laminectomy area. In our study, we observed that the fat tissue generated gradually in the ECM+aMSCs groups. No obvious fat signal was observed at $4^{\text {th }}$ week and the high-density signal indicating formation of fat tissue was observed between the epidural sac and epidural scar tissue at $12^{\text {th }}$ week in the ECM+aMSCs groups. At the same time, a gradual increase of the epidural scar tissue signal was observed in each group after laminectomy. In consistence with MRI observation, the fat tissue around epidural in the ECM+aMSCs group and the adherence of the epidural scar and the dura matar in the control group were observed via Oil staining at $12^{\text {th }}$ week. On the contrary, no evident epidural space was observed in either the control group or the ECM gel group at the $12^{\text {th }}$ week from MRI, which suggested the adherence of fibrous tissue and the dural sac. Taken together, these results demonstrated that we could reconstruct the epidural fat tissue between the fibrous tissue and the dural sac via the adipose Tissue Engineering.

The proliferation and extracellular matrix production of fibroblasts are important to the formation of fibrosis ${ }^{18}$. Wan Qi et al. ${ }^{19}$ found that inhibition of fibroblast proliferation could reduce surgery-induced epidural fibrosis in SD male rats. IL- 6 and TGF- $\beta$ play an important role in the generation of fibrosis, and epidural fibrosis can be prevented via downregulation of the TGF- $\beta$ and IL-6 in laminectomy ${ }^{5,20-22}$. In our study, The number of fibroblasts showed a trend of increasing first and then reducing in each group by H\&E staining, the control group and the ECM gel group reached the highest at the 8th week, and the ECM+aMSCs group at the 12th week. The ratio of fibrosis protein increased in each group as identified by Masson's trichrome staining, but the ECM gel group and ECM+aMSCs groups were lower than the control group. Meanwhile, the relative expression of TGF- $\beta$ and IL- 6 was inhibited at the $4^{\text {th }}$ week $(P<0.05)$, and higher expression was observed in the ECM gel group and ECM +aMSCs group at later stage. This might be because the ECM gel caused local inflammation during degradation. These results suggested that 
epidural fibrosis was inhibited in the ECM gel group and ECM+aMSCs group at the early stage via inhibiting the expression of inflammatory factor IL- 6 and fibrosis factor TGF- $\beta$.

Although epidural fat reconstructed between the dural sac and epidural fibrous tissue was observed by MRI and Oil Red $O$ staining at the $12^{\text {th }}$ week, and epidural fibrosis was inhibited as shown by the Masson's trichrome staining, there was no significant difference between the ECM gel group and ECM+aMSCs group for TGF- $\beta$ and IL- 6 at the $4^{\text {th }}$ and $8^{\text {th }}$ week. We only used three rabbits in each check point, our results may have been due to chance, the results must be confirmed in a larger study. Besides acting as seed cells in the adipose tissue-engineering, is it the role of aMSCs in inhibited the inflammatory response may need more research.

\section{Conclusion}

In this study, we identified that adipose tissue engineering could reconstruct the epidural fat as a physiological barrier, thereby reducing the local inflammatory factors at the early stage and inhibiting epidural fibrosis. Thus, the tissue-engineered fat may provide a feasible solution for clinical prevention of epidural fibrosis adhesion after laminectomy, and the successful construction of epidural fat may give a long-term satisfaction for the laminectomy patients without FBS.

\section{Declarations}

\section{Funding}

This study was supported by the Foundation of Shanghai Municipal Commission of Health and Family Planning (Grant No. 20184Y0208), by the China Scholarship Council, and by the High-level Talents Special Fund of the Minghang District of Shanghai, China. No benefits in any form have been or will be received from a commercial party related directly or indirectly to the subject of this manuscript.

\section{Availability of data and supporting materials}

The datasets supporting the conclusions of this article are included within the article and its supplementary materials.

\section{Authors' contributions}

Xiangsheng Liu and Feifei Zhang performed the whole experiments and were responsible for the all data and drafting of the article. Youhai Dong designed the study and contributed to the preparation of the manuscript. Linli Li and Yiqun He helped in the performance of animal surgeries and the interpretation of data. All authors read and approved the final manuscript.

\section{Competing interests}

All authors declare that they have no competing interests. 


\section{Consent for publication}

Not applicable.

\section{Ethics approval}

The operation on New Zealand rabbits in this experiment was approved by the Fudan University Ethics Committee and the Institutional Review Board of the Fudan University School of Medicine.

\section{Acknowledgments}

We would like to thank Xiaojie Han for her excellent work.

\section{References}

1. Avellanal M, Diaz-Reganon G, Orts A, Gonzalez-Montero L, Riquelme I. Transforaminal Epiduroscopy in Patients with Failed Back Surgery Syndrome. Pain Physician. 2019;22(1):89-95

2. Sun Y, Zhao S, Li X et al. Local application of rapamycin reduces epidural fibrosis after laminectomy via inhibiting fibroblast proliferation and prompting apoptosis. J Orthop Surg Res. 2016;11(1):58

3. Dai J, Li X, Yan L et al. The effect of suramin on inhibiting fibroblast proliferation and preventing epidural fibrosis after laminectomy in rats. J Orthop Surg Res. 2016;11(1)

4. Meng XM, Nikolic-Paterson DJ, Lan HY. TGF-beta: the master regulator of fibrosis. Nat Rev Nephrol. 2016;12(6):325-38

5. Lichtman MK, Otero-Vinas M, Falanga V. Transforming growth factor beta (TGF-beta) isoforms in wound healing and fibrosis. Wound Repair Regen. 2016;24(2):215-22

6. Su C, Sui T, Zhang X, Zhang H, Cao X. Effect of topical application of mitomycin-C on wound healing in a postlaminectomy rat model: An experimental study. Eur J Pharmacol. 2012;674(1):7-12

7. Sun $Y$, Wang $L X$, Wang $L$ et al. A comparison of the effectiveness of mitomycin $C$ and 5 -fluorouracil in the prevention of peridural adhesion after laminectomy. J Neurosurg Spine. 2007;7(4):423-8

8. Isık S, Taşkapılıoğlu MÖ, Atalay FO, Dogan S. Effects of cross-linked high-molecular-weight hyaluronic acid on epidural fibrosis: experimental study. Journal of Neurosurgery: Spine. 2015;22(1):94-100

9. Rabb CH. Failed back syndrome and epidural fibrosis. The Spine Journal. 2010;10(5):454-455

10. Da Costa RC, Pippi NL, Graça DL et al. The effects of free fat graft or cellulose membrane implants on laminectomy membrane formation in dogs. The Veterinary Journal. 2006;171(3):491-499

11. Choi HJ, Kim KB, Kwon Y. Effect of Amniotic Membrane to Reduce Postlaminectomy Epidural Adhesion on a Rat Model. J Korean Neurosurg S. 2011;49(6):323

12. Li L, Chen X, He Y, Dong Y. Biological and Mechanical Factors Promote the Osteogenesis of Rabbit Artificial Vertebral Laminae: A Comparison Study. Tissue Eng Part A. 2018;24(13-14):1082-1090 
13. Xu J, Chen Y, Yue Y, Sun J, Cui L. Reconstruction of epidural fat with engineered adipose tissue from adipose derived stem cells and PLGA in the rabbit dorsal laminectomy model. Biomaterials. 2012;33(29):6965-6973

14. Dong $Y$, Chen $X$, Wang $M$, Hong Y. Construction of artificial laminae of the vertebral arch using bone marrow mesenchymal stem cells transplanted in collagen sponge. Spine (Phila Pa 1976). 2012;37(8):648-53

15. Liu FB, Lin Q, Liu ZW. A study on the role of apoptotic human umbilical cord mesenchymal stem cells in bleomycin-induced acute lung injury in rat models. Eur Rev Med Pharmacol Sci. 2016;20(5):969-82

16. Iwatani S, Yoshida M, Yamana K et al. Isolation and Characterization of Human Umbilical Cordderived Mesenchymal Stem Cells from Preterm and Term Infants. Journal of visualized experiments : JoVE. 2019;(143)

17. Sobti S, Grover A, S John B, Grewal S, George U. Prospective randomized comparative study to evaluate epidural fibrosis and surgical outcome in patients undergoing lumbar laminectomy with epidural autologous free fat graft or gelfoam: A preliminary study. International Journal of Applied and Basic Medical Research. 2018;8(2):71

18. Jin H, Wang Z, Gu Z et al. Schisandrin B attenuates epidural fibrosis in postlaminectomy rats by inhibiting proliferation and extracellular matrix production of fibroblasts. Phytother Res. 2019;33(1):107-116

19. Wan Q, Chen H, Li X et al. Artesunate inhibits fibroblasts proliferation and reduces surgery-induced epidural fibrosis via the autophagy-mediated p53/p21waf1/cip1 pathway. Eur J Pharmacol. 2019;842:197-207

20. O'Reilly S, Ciechomska M, Cant R, van Laar JM. Interleukin-6 (IL-6) trans signaling drives a STAT3dependent pathway that leads to hyperactive transforming growth factor-beta (TGF-beta) signaling promoting SMAD3 activation and fibrosis via Gremlin protein. J Biol Chem. 2014;289(14):9952-60

21. Rani N, Bharti S, Bhatia J et al. Inhibition of TGF- $\beta$ by a novel PPAR-y agonist, chrysin, salvages $\beta$ receptor stimulated myocardial injury in rats through MAPKs-dependent mechanism. Nutr Metab. 2015;12(1):11

22. Zhang C, Kong X, Zhou H et al. An Experimental Novel Study:Angelica sinensis Prevents Epidural Fibrosis in Laminectomy Rats via Downregulation of Hydroxyproline, IL-6, and TGF- $\beta 1$. Evid-Based Compl Alt. 2013;2013:1-7

\section{Table}

\section{Table 1. the sequence of primer}


'- $\beta$ 5'- AAC GGG CTC AAC ATC TAC AC-3' 5' -GGT CCT TGC GGA AGT CAA T-3'

5' -TTC CAA GGC TGA TAG AAG AA -3' 5' -CAG GCA GGT CTC ATT ATT CA-3'

tin 5' CCC GAC AGC CAG GTC ATC-3'

5' GTT GAA GGT GGT CTC GTG GA-3'

Figures

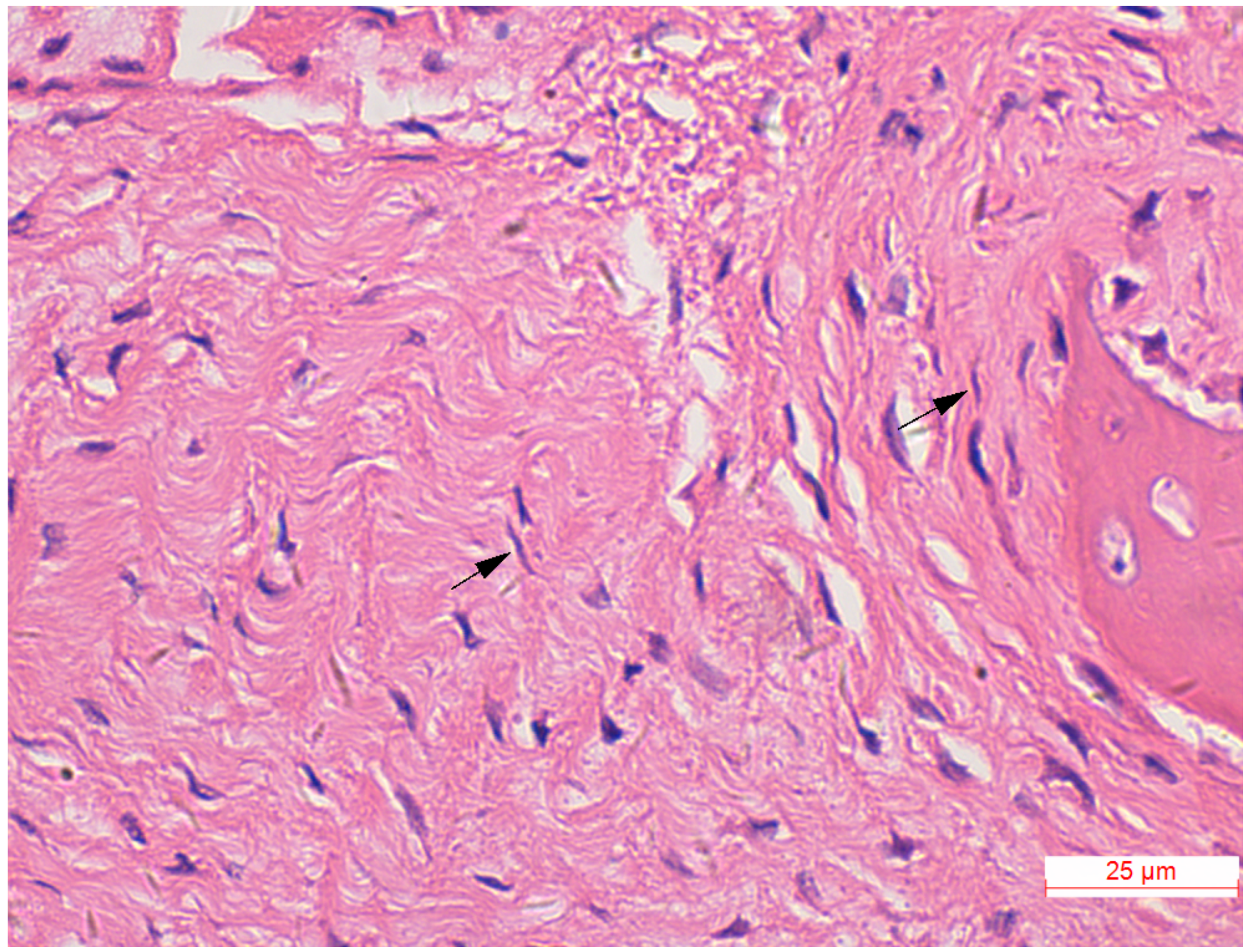

\section{Figure 1}

The number of fibroblasts was counted under 400 -fold magnification(black arrow shows the fibroblasts ). 


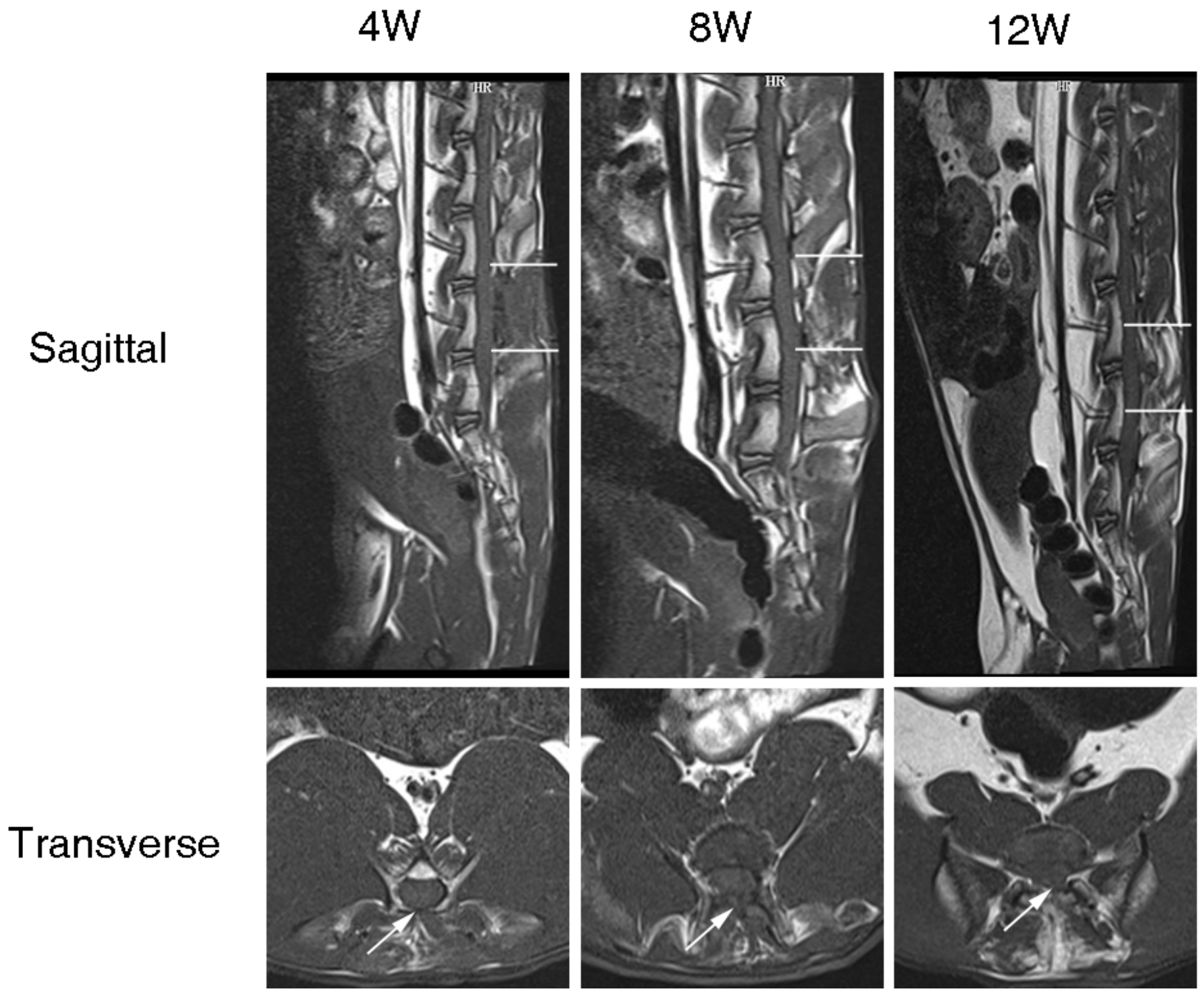

Figure 2

The images of MRI of the laminectomy site in control group at 4th ,8th and 12th weeks. The arrow head show the space between dura mater and epidural scar tissue. The dashed line enclose area in the sagittal images show the laminectomy site. 


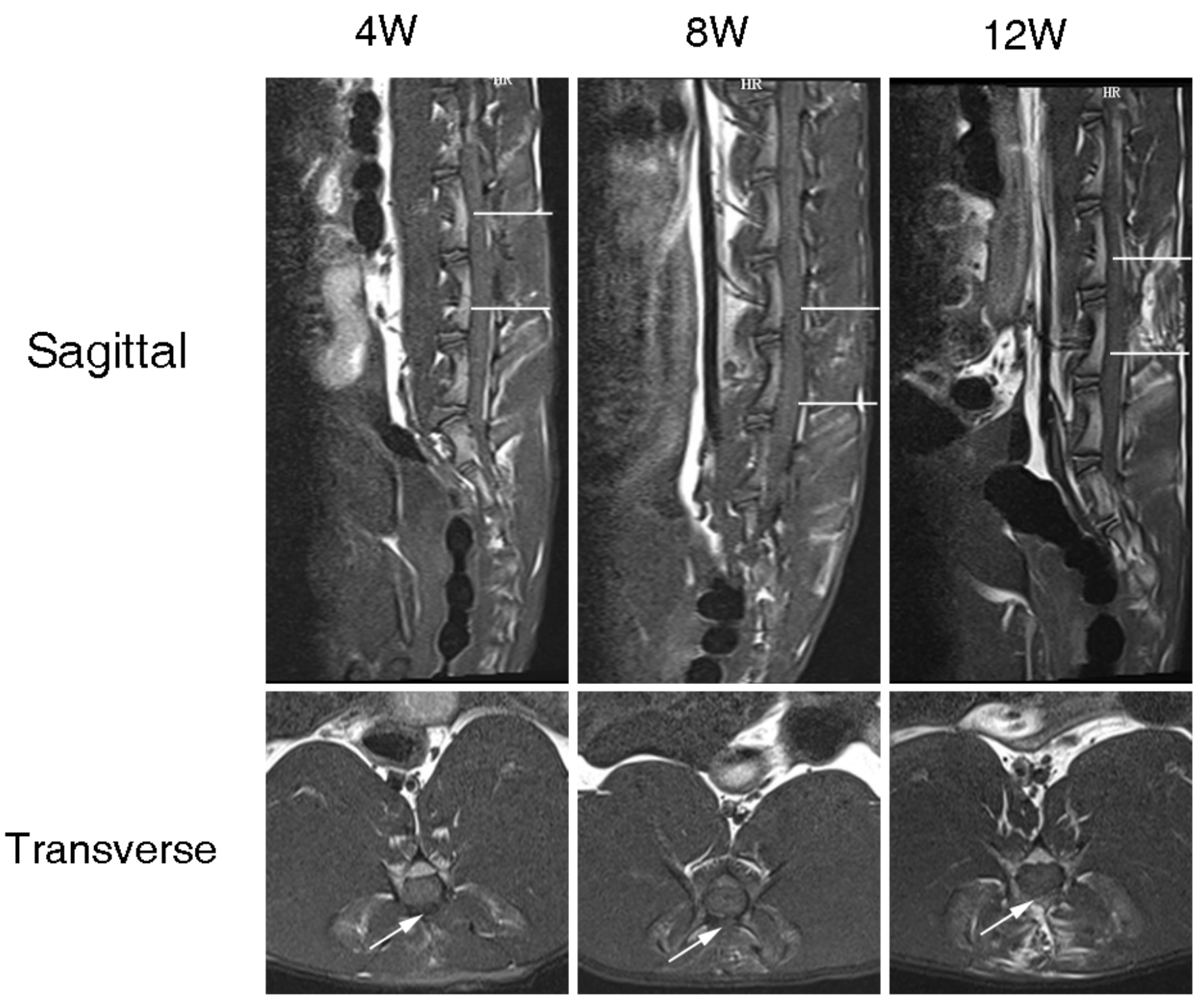

Figure 3

The images of MRI of the Laminectomy site in ECM gel group at 4th ,8th and 12th weeks. The arrow head show the space between dura mater and epidural scar tissue. The dashed line enclose area in the sagittal images show the laminectomy site. 


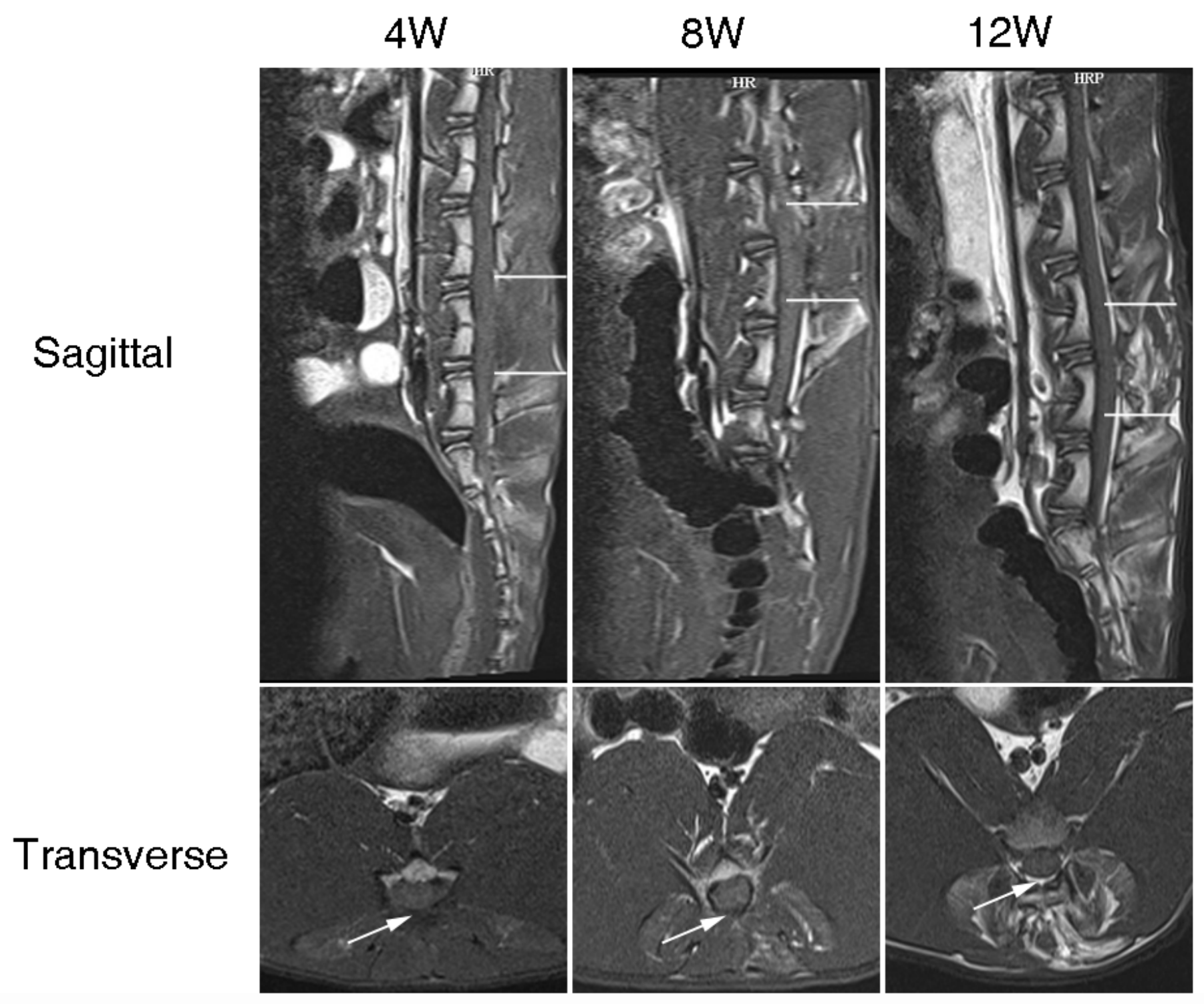

Figure 4

The images of MRI of the laminectomy site in ECM gle+cells group at 4th ,8th and 12th weeks. The arrow head show the space between dura mater and epidural scar tissue. The dashed line enclose area in the sagittal images show the laminectomy site. 

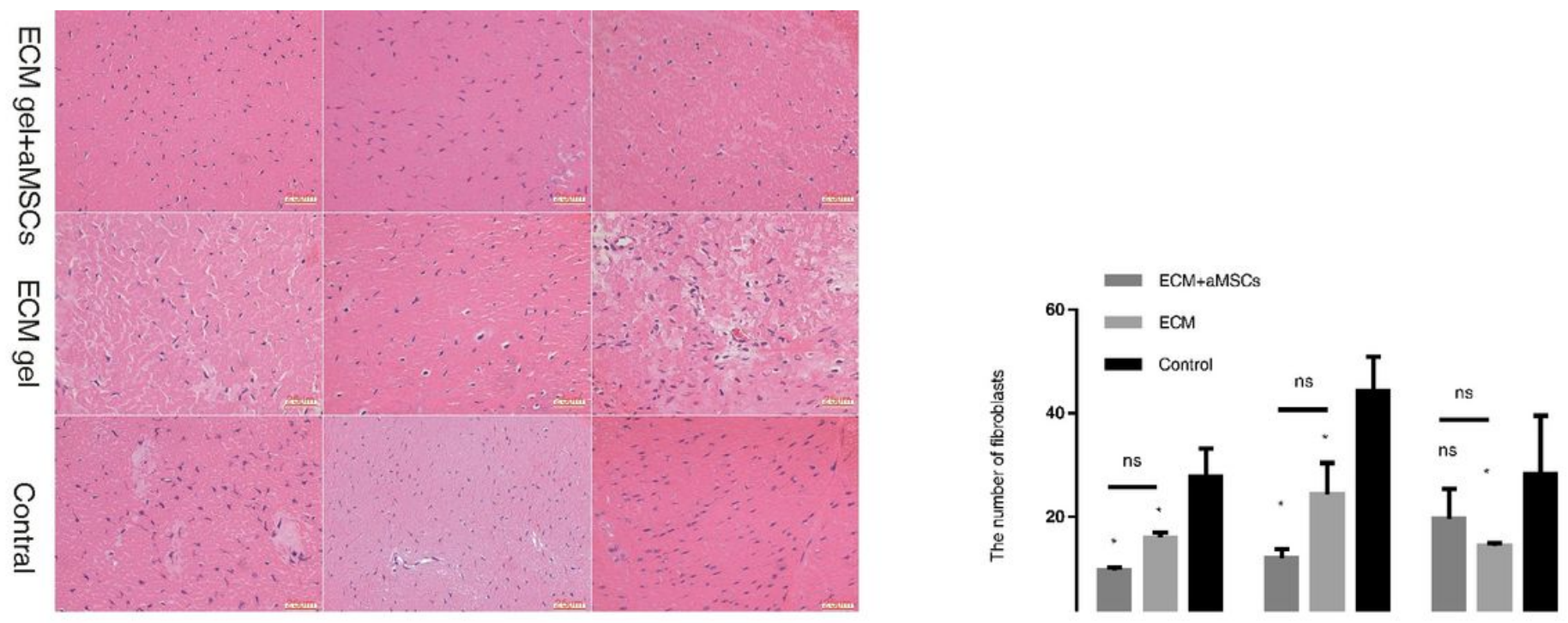

\section{Figure 5}

H\&E staining of the laminectomy area under 400 -fold magnification. (a)shown the fibroblasts in each group and each time under the 400 -fold magnification.(b) the number of fibroblasts was counted at $400-$ fold high magnification. ${ }^{*} \mathrm{P}<0.05$ compared with the control group, and \# $\mathrm{P}<0.05$ between the two groups, and ns mean $\mathrm{P}>0.05$. Scal bars $=25 \mu \mathrm{m}$
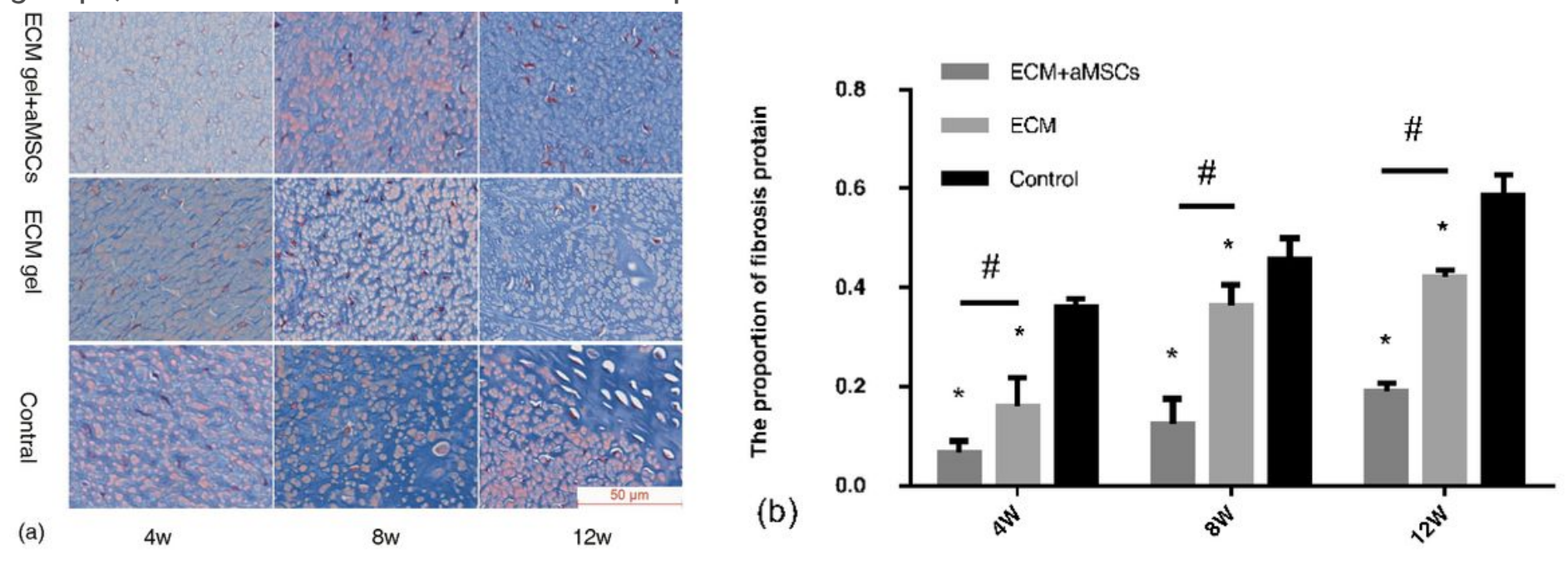

\section{Figure 6}

Masson's trichrome staining of the laminectomy area.(a)the blue in the figure shows the collagen fibers . (b) Statistically analyze of the percentage of collagen fibers under 200 -fold high magnification. ${ }^{\mathrm{P}}<0.05$ compared with the control group, and \# $\mathrm{P}<0.05$ between the two groups, and ns mean $\mathrm{P}>0.05$.Scal bars $=50 \mu \mathrm{m}$. 


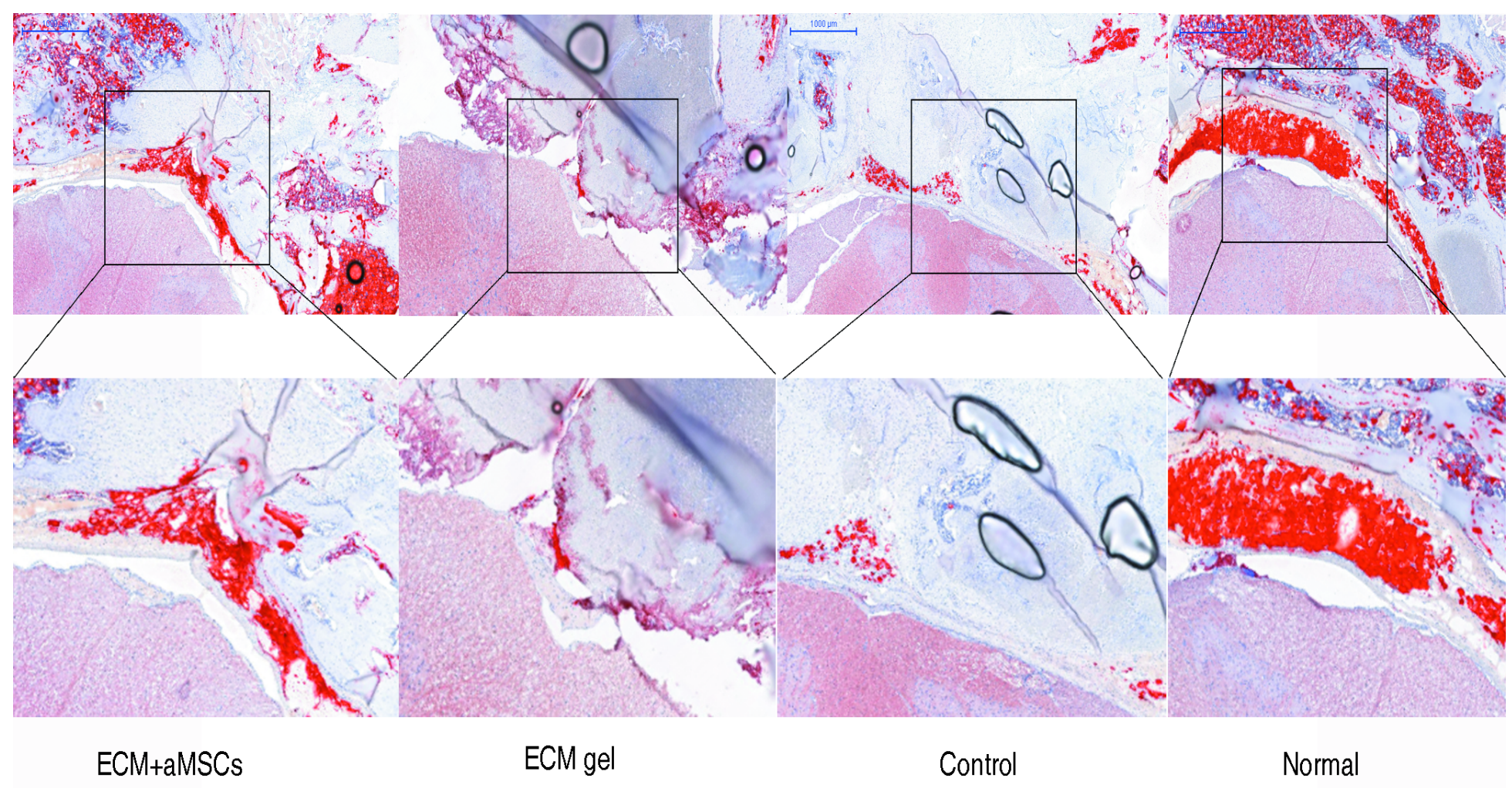

Figure 7

The Oil Red 0 staining show the epidural fat at 12th week, the red show the fat.
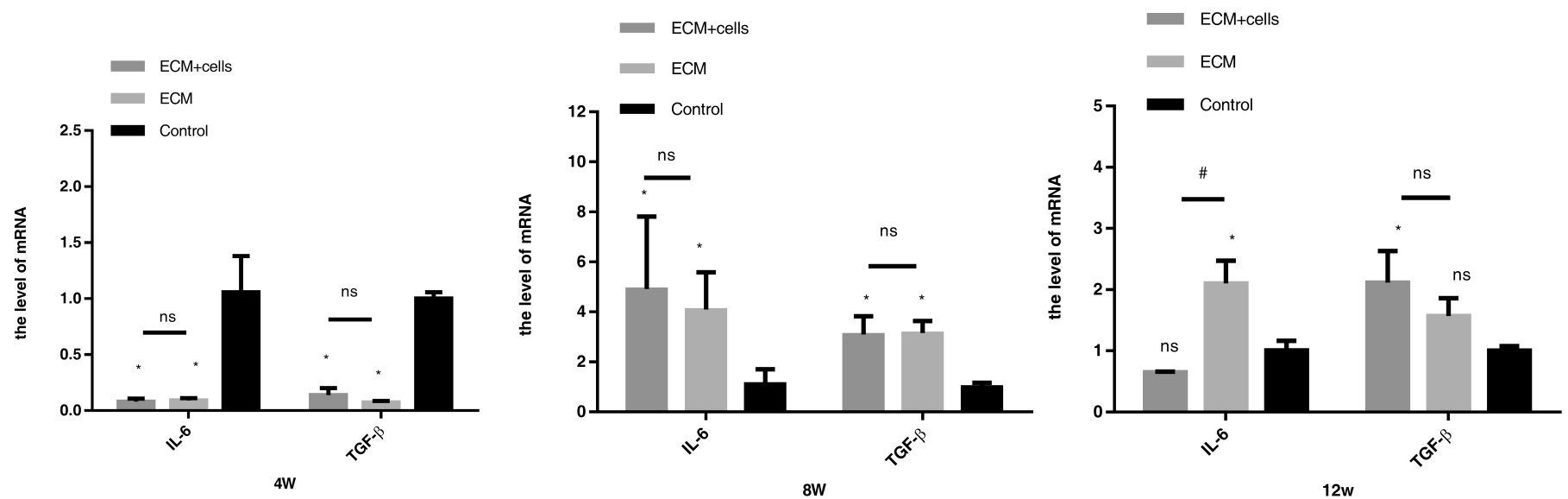

8W

$12 w$

\section{Figure 8}

QRT-PCR was used to detect the relative expression of inflammatory factors of IL- 6 and TGF- $\beta$. *P $<0.05$ compared with the control group, and \# $\mathrm{P}<0.05$ between the two groups, and ns mean $\mathrm{P}>0.05$. 